\title{
LA SALVE REGINA EN LAS DOCTRINAS CRISTIANAS Y CARTILLAS DEL S. XVI
}

\author{
MARGHERITA MORREALE \\ Universidad de Padua
}

La salve o salve regina como oración se inspira en la intercesión de la Virgen, a quien Cristo desde la cruz encomendara el género humano, según la interpretación de Jn 19,27 «Ecce mater tua», que pasó a la liturgia como «nosque... in cruce pendens Matri Virgini commendasti ${ }^{1}$. Por expresar esta intercesión con absoluta certeza y de modo entrañable es la plegaria mariana que más aceptación ha obtenido entre los fieles. Ya en el s. XIII en el Setenario de Alfonso el Sabio se lee: «Santa María tiene tendidos los sus braços para recebir e perdonar los peccadores, e averles mercet, rogando a Dios por ellos» ${ }^{2}$, $\mathrm{y}$, más adelante: «La Virgen María es más cerca de los peccadores e de los cuitados que los otros santos para oírlos y para acorrerlos seyendo auogada entre Dios e ellos» (pág. 81). De la solemnidad a la par que de la hermosura de la salve da fe Dante Alighieri en el canto VII del Purgatorio dedicado a los príncipes y grandes señores en los versos 82-83,

Salve, Regina, in sul verde e in sui fiori quivi seder cantando anime vidi.

En latín, como antífona pertenece a la liturgia ${ }^{3}$ (Pedro Simón Abril [v.i.] la llamaba «Salutación de la santa Iglesia a la santíma Virgen»), y acusa un tenor

${ }^{1}$ Cf. A. Blaise, Le vócabulaire latin des principaux thèmes liturgiques (Tumhout, 1966), al que remitiremos también para las citas biblicas corrientes para considerarlas en su entorno lituírgico; aquí interesa especialmente el capítulo VIL «Prières à la sainte Vierge»; vid. \& 116; para una ulterior documentación y eventuales remites al latín clásico, véase del mismo autor, Dictionnaire Latin-français des auteurs chrétiens (ib., 1954); a éstos nos referiremos con Blaise y Blaise . $_{2}$.

2 Ed. K. H. Vanderford, Buenos Aires, 1945, págs. 100-101.

3 Para más precisión citamos a A. Ruiz Gutiérrez en el artículo dedicado a la Salve regina en la Gran Enciclopedia Rialp, Madrid, 1971-87: «Se reza durante todo el ciclo de Pentecostés, desde el sábado antes de la fiesta de la Trinidad hasta el sábado antes del primer domingo de

RFE, LXXXIV, 2004, 1. , págs. 129-151 
constante desde hace un milenio, con las inevitables variantes. De esta duración milenaria ${ }^{4}$ se sabe más que de la autoría ${ }^{5}$. Junto con el Ave María es una de las oraciones más imitadas en himnos latinos, entre ellos en uno atribuido a San Buenaventura ${ }^{6}$.

La salve se abre como la que más a las melodías, sospechamos que saben más de la salve los cultores de la música ${ }^{7}$ que los filólogos de nuestro texto, siquiera del vernáculo, pero los instrumentos que tenemos a la mano, aun los más divulgados, nos permitirán examinarlo y compararlo con la matriz latina. Del texto se nos dice que representa una prosa rítmica que puede disponerse del modo siguiente ${ }^{8}$ :

1. Salve, Regina / Mater misericordiae,

2. Vita, dulcedo / et spes nostra, salve!

3. Ad Te clamamus / exules filii Hevae

4. Ad Te suspiramus / gementes et flentes In hac lacrimarum valle.

5. Eia ergo, / advocata nostra, Illos tuos misericordes oculos / ad nos converte

6. Et lesum, / benedictum fructum ventris tui, Nobis post hoc exilium ostende.

7. O Clemens, /o pia, O dulcis / Virgo Maria,

ello rimando en $e: 1$. misericordie, 2. salve, 3. Heve, 4. valle, 5. converte, 6 . ostende, con los dos últimos versos como quinarios en $a$ : 7. pia: Maria.

Al final el responsorio de la antífona reza: «Ora pro nobis, sancta Dei genitrix, ut digni efficiamur promissionibus Christi».

Adviento; al ampliarse ese ciclo y recibir la denominación de tiempo per annum, a partir de 1970, se reza también en el tíempo comprendido entre el final del ciclo de Navidad (Epifanía) y el comienzo del de Cuaresma (miếrcoles de Ceniza)»; esto aparte del rezo en el oficio divino. La otra vertiente en la que no hemos podido entrar sería la que sugiere en parte el escrito citado por el mismo, J. M. Canal, Salve Regina Misericordiae. Historia y leyendas en tomo a esta antifona, Roma, 1963.

4 Fue introducida en 1135 en la liturgia de Cluny y luego aceptada por los cistercienses y más tarde por los dominicos, que hasta hoy la rezan diariamente, después de completas; para más particularidades y bibliograffa, cf. I. Cecchetti en Enciclopedia Cattolica (Città del Vaticano, 1953), s.v.

s Habiéndose excluido la de San Bernardo, por más tardfo, se sugiere la del monje de Reichenau, Hermannus Contractus (1013-54), llamado así por su cojera, quien a pesar de sus defectos físicos (a penas podía levantar la voz) dejó farna de maestro y de bombre de saber universal; o la de Pedro, obispo de Compostela († 1000) (cf. M. Rubén García Álvarez, San Pedro de Mesonzo, el origen y el autor de la «Salve Regina», Madrid, 1965, habiéndose eliminado al pa-

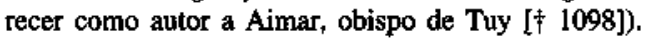

6 F. Daniel, Thesaurus hymnologicus, Leipzig, 1855-56, vol. 2, págs. 323-26.

${ }^{7}$ Cf. J. Pothier, «Antienne Salve Regina», Revue du Chant Grégorien, 10, 1902, 145-52.

${ }^{8}$ Segín I. Cecchetti, loc. cit. 
Los documentos que hemos de examinar son, una vez más, las Doctrinas y Cartillas en las que se cita la salve entre «las oraciones que el cristiano es obligado a saben» (cf. Flórez: «y assí pienso que la deuéis bien de saber» fol. lviiiv); o sea: las siguientes, que citaremos según las reproducciones facsimilares de V. Infantes, con la numeración y las fechas de éste ${ }^{9}$, con intercalada la Doctrina cristiana del P. Flórez según la edición también facsimilar de P. M. Cátedra ${ }^{10}$ :

(I) Hemando de Talavera, Breve doctrina (c. 1496) ${ }^{11}$; (II) f́d., Cartilla y doctrina (c. 1501-1508); (III) Cartilla para mostrar a leer (c. 1526); (V) Hernando de Talavera, Breve doctrina (c. 1539); (VIII) Francisco Falero, Cartilla para enseñar a leer (1545); (IX) Cartilla y Doctrina Christiana para que deprendan los niños (1549); Flórez, 1552 (no está documentada la $1^{\mathrm{a}}$ impresión de 1546); (X) Juan de Ávila, Doctrina Christiana que se canta (1558, según el frontispicio; 1554 en la lista de Infantes ${ }^{12}$ ); (XI) íd., 1558; (XII) Juan de Robles, Arte para enseñar muy breve (c. 1564); (XVI) Martín de Ayala, Doctrina Christiana (1566), (XVII) Pedro de Gante, Cartilla para enseñar a leer nuevamente enmendada (1569), (XXI) Francisco de Navarra, Cartilla y breu instructió (1571), (XXII) Cartilla para enseñar a leer (c. 1573), (XXIII) Cartilla y Doctrina Christiana (1574), (XXIV) Juan de Ávila, Doctrina Christiana que se canta (1574), (XXV) A a b c Cartilla para mostrar a leer (1576), (XXVI), Cartilla para mostrar a leer (1577), muy parecidas, (XXVII) Cartilla y Doctrina Christiana (1584), igual a la anterior, excepto por el frontispicio; (XXXI) Cartilla y Doctrina Christiana (1588); (XXXII) Pedro Simón Abril, Instrucion para enseñar a lo niños facilmente el leer y el escrivir, 1590; Ripalda, (XXXIII) Cartilla para enseñar a leer a los niños $\left(1596=1606^{13}\right)$.

Divididas por idioma presentan la salve solo en castellano las siguientes: (I) c. 1496, (II) c. 1501-1508, (V) 1539; (X-XI) 1554, 1558 (XXXII) Pedro Simón Abril, Ripalda 1591; en latín y castellano, éstas: (III) 1526, (VIII) 1545, Florez fol. lxxiiij y fol lix; (XII) 1569, (XXII) 1573 (con el texto latino incompleto por un corte de parte de la hoja), (XXV) 1576; (XXVI) 1577; (XXVII), 1584; solo en latín ésta otra: (XII) 1564, en castellano y «lengua aráuiga» ésta: (XVI) 1566; en latín, castellano y lengua amerindia izcatqui ésta: (XVII) 1569.

\footnotetext{
9 Véase Víctor Infantes, De las primeras letras. Cartillas españolas para enseñar a leer de los siglos XV y XVI. Preliminar y edición facsímil de 34 obras, Salamanca, Universidad, 1998.

10 Cf. La Doctrina Cristiana del ermitaño y niño de Andrés Flórez, O. P., Valladolid, 1552.

11 Del Arzobispo de Granada, Fr. Hernando de Talavera, citaremos también del Tratado de lo que significan las ceremonias de la Misa, en Nueva Biblioteca de Autores españoles (en adelante NBAE), vol. 16, Madrid, 1911, págs. 73-93.

12 La confusión puede ser debida a la pluralidad de ediciones de la que gozó el catecismo de Juan de Ávila.

13 Sobre ésta reconstruye Infantes la que aquí interesa para la edición perdida de 1596.
} 
Contienen además de la salve el versículo del responsorio las siguientes: (I-II-V) 1496, c. 1501-1508, c. 1539; el responsorio entero, todas las otras.

Solo consideraremos el texto latino y el casteliano, que reproducimos en sus distintas realizaciones, porque nos han de interesar en el aspecto tipográfico para la historia de la imprenta, y en el verbal del vernáculo por lo que delatan de la evolución paulatina del castellano antes de su última fijación, como ya vimos acerca del padrenuestro ${ }^{14}$, y más por cuanto la salve ilustra a las claras la convivencia de la lengua vernácula con la latina. Los textos, reproducidos verbatim son los siguientes, empezando por los latinos:

(III) 1526, (XII) c. 1564 y (XV) 1566 (Becerol, sin responso) en latín, con las diferencias intercaladas:

SAlue regina mater misericordia [XII XV misericordie] [XII XV.] vita [XI $\mathrm{XV}$ V] dulcedo $\tau$ [XII et] [XV Ø] spes nostra [XII XV nostra] salue [XV saue]. [XV Ø] Ad te clamamus [XII clamamus] exules filii eue. [XV Ø] Ad te suspiramus gementes $\tau$ [XV et] flentes [XII XV flentes] in hac lachrimarum valle. [XV Ø] Eya ergo [XII r go] aduocata nostra [XII XV :] illos tuos misericordes oculos ad nos conuerte [XII converte]. Et iesum [XII XV J] bendictum [XII XV benedictum] fructum [XII XV fructum] ventris tui [XII.] nobis [XII N] post hoc exilium [XII XV exilium] ostende [XII ostende]. O clemens [XI clemens] [XII .] o [XII O] pia [XII .] o [XII XV O] dulcis virgo [XV virgo] [XII semper virgo] maria [XI XV M] $[\mathrm{XV}$.] $[\mathrm{XII} \mathrm{XV}$ Amen]. v/. Ora pro nobis sancta [XII sancta] dei genitrix. R/. Ut digni efficiannur promissionibus xpi [XII Christi].

\section{Flórez 1552:}

I Salue regina mater misericordie. Vita dulcedo. Spes nostra salue. Ad te clamamus. Exules filii Eue, ad te suspiramus, gementes et flentes, in hac lachrymarum valle. Eya ergo aduocata nostra. Illos tuos misericordes oculos ad nos conuerte. Et lesum benedictum fructum ventris tuy : nobis post hoc exilium ostende. $O$ clemenes [sic]. $O$ pia. $O$ dulcis virgo Maria. Ora pro nobis sancta dei genitrix. Ut digni efficiamur promissionibus Christi. Amen Jesus, fol. lix v y r.

En castellano, empezando por la Cartilla (I) 1496:

SAlue reyna de misericordia. Dulçor de vida. Esperança nuestra Salue. Ati clamamos desterrados hijos de eua. Ati suspiramos gemiendo y llorando en este lloroso valle. Ea pues abogada nuestra aquellof tus misericordiosos ojos a nos buelue. Y a jesu cristo bendicto fruto de tu vientre: despues deste destierro a nos demuestra. $O$ clemente: o pia. $O$ dulce virgen maria Ruega por nos sancta madre de dios,

14 Véase en «El padrenuestro en las Doctrinas cristianas del s. XVI», en I quattro universi $d t$ discorso, Atti del Congresso Internazionale «Orationis Millennium» L'Aquila, 24-30 giugno 2000, ed. Giuseppe de Gennaro S.I., Città del Vaticano, 2002, págs, 432-89. 
La comparación entre la Cartilla (I) y la (II) arroja lo siguiente:

(I-II-V) 1496, c. 1501-1508, c. 1539:

SAlue reyna de misericordia, Dulçor de [V Ø] vida. Esperança [V n] nuestra [II .] Salue [V s]. A ti clamamos desterrados hijos de eua [V E]. A ti suspiramos gemiendo [V gimiendo] [II $n$ ] y [II $\tau$ ] llorando [V I] en este lloroso valle. Ea pues abogada [II auogada] nuestra aquellos tus misericordiosos ojos a nos buelue. Y [II E] a jesu [II iesu] cristo [V christo] bendicto [II V bendito] fruto [V fructo] de tu vientre [II $n$ ]: [II $\emptyset][V$.] despues deste destierro a nos demuestra [II demuestra]. O clemente: [II Ø] [V .] o [II O] [V O] pia. O dulce virgen [II $n$ ] maria [II .] Ruega por nos sancta [II santa] madre de dios.

Prosiguiendo con las Cartillas (III) 1526 y (VIII) 1545:

SAlue te dios reyna madre de misericordia: vida y [VIII $\emptyset$ ] dulçura y esperança nuestra [VIII .] dios [VW D] te salue. A ti llamamos los desterrados hijos de Eua. A ti sospiramos gimiendo y llorando [VIII n] en aqueste valle de lagrimas. [VIII :] Ea [VIII ea] pues abogada nuestra buelue a nosotros essos tus misericordiosos [VIII se] ojos : [VIII .] y [VIII Y] despues de [VIII de] aqueste destierro muestra nos a Jesu bendito [VIII $\mathbf{n}$ ] fruto [VIII fructo] detu [VIII de tu] vientre [VIII $\mathrm{n}$ ] [VIII .] despues [VIII Ø] que [VIII Ø] deste [VIII Ø] destierro [VIII Ø] seremos [VIII Ø] salidos [VIII Ø] $O$ mansa [VIII clemente]. O piadosa $O$ dulce virgen [VIII virgen] maria [VIII Maria]. v/. Ruega por nos sancta [VIII sancta] madre de [VIII de] dios [VIII.] R/. Que seamos dignos delas promissiones [VIII promisiones] de christo. Amen.

\section{En Flórez 1552 el texto suena:}

I Dios te salue reyna madre de misericordia Dulçor. Vida. Esperança nuestra. Dios te salue. A ti llamamos los desterrados hijos de Eua. A ti sospiramos, gimiendo, y llorando en este valle de lagrimas. Ea pues que assi es (sic) abogada nuestra: buelue a nosotros tus ojos de misericordia. $Y$ despues deste destierro: muestranos a Jesus fructo bendito de tu vientre. $O$ clemente. $O$ piadosa. $O$ dulce virgen Maria. Ruega por nos virgen madre de dios. Porque seamos dignos de los prometimientos de Jesu cristo. Amen Jesus. fol. lxxiiij $\mathbf{r}$ y v.

Las dos siguientes (X) 1554 y (XI) 1558 (a dos columnas) se presentan así, con el $[\emptyset]$ para señalar la ausencia de los signos de puntación, sin correspondencia en la distribución de las columnas: 


II [XI Ø] DIos te salue
reyna [XI R] y madre
de misericordia. [XI $\emptyset]$
Vida $[\mathrm{XI}$ v] / [XI $\emptyset]$ y dulçura /
$\quad[\mathrm{XI} \emptyset]$
esperança nuestra. [XI $\emptyset]$
Dios te salue. [XI $\emptyset]$
A $[\mathrm{XI}$ a] ti llamarnos
los desterrados
hijos de Eua. [XI $\emptyset]$
A [XI a] ti sospiramos
gimiendo y llorando
en este valle
de lagrimas. [XI $\emptyset]$
Ea pues
aduogada $[X I$ abogada] nuestra
buelue a nos

essos [XI aquessos] tus ojos misericordiosos. [XI Ø] $\mathrm{Y}[\mathrm{XI} \mathrm{y}]$ despues

deste [XI deste] destierro muestra nos a jesus [XI J] fructo bendito [XI bendicto] del [XI de] ta vientre. [XI Ø] $O$ clementissima $\mathrm{O}$ [XI o] piadosa. [XI Ø] $\mathrm{O}$ [XI o] dulce siempre virgen $[\mathrm{XI} n]$ maria $[\mathrm{XI} \mathrm{M}]$ Ruega [XI r] por nos sancta madre de dios [XI D]. [XI Ø] Porque [XI porque] seamos dignos delos [XI de los] prometimientos [XI n] de jesu [XI J] christo [XI C]. Amen [XI Amen].

Proseguimos con las otras a renglón seguido:

(XVI) 1566:

DIos te salue o Reyna, madre de misericordia, vida, dulçura, esperança nuestra, Dios te salue. Ati llamamos los desterrados hijos de Eua a ti sospiramos, gimiendo, y llorando en este valle de lagrimas. Ea pues abogada nuestra, buelue a nosotros essos tus ojos misericordiosos y a Iesus fructo bendito de tu vientre, muestra nos le despues deste destierro. $O$ clemente, $O$ piadosa, o dulce Maria virgen. Ruega por nos sancta madre de Dios, que seamos hechos dignos de las promessas de Christo Iesu. Amen.

(XVII) 1569:

SAlue te Dios reyna y madre de misericordia, vida dulçura y esperança nuestra Dios te salue. A ti llamamos los desterrados hijos de Eua. A ti sospiramos gimiendo y llorando en aqueste valle de agrimas [sic]. Eya pues abogada nuestra buelue a nosotros essos tus misericordiosos ojos. Y despues de aquel ste [sic] destierro muestranos a Jesu bendito fructo de tu vientre. $O$ clemente. $O$ piadosa. $O$ dulce virgen Maria $v /$. Ruega por nos sancta madre de dios. R. Que seamos dignos de las promissiones de Christo. Amen.

(XXII) 1573:

DIos te salue, reyna y madre de misericordia, vida, dulçor, y esperança nuestra, salue te Dios Ati llamamos los desterrados hijos de Eua, a ti sospiramos gimiendo y llorando en este valle de lagrimas. Pues ya abogada nuestra, buelve a nosotros aquellos tus ojos de misericordia, y muestra nos a Jesu bendicito fructo de ta vientre despues que deste destierro seremos salidos. O mansa, o piadosa, o misericordiosa Maria. Ruega por nos sancta madre de Dios. $\mathrm{R}$. Porque seamos dignos delas promissiones $\mathrm{d} e$ Christo. 


\section{(XXV) 1576:}

SAlue te Dios Reyna y madre de misericordia, vida y dulçura esperança nuestra. Dios te salue a ti llamamos los desterrados hijos de Eua. A ti sospiramos gimiendo y llorando en aqueste valle de lagrimas. Ea pues abogada nuestra buelue a nosotros essos tus misericondiosos ojos, y despues de aqueste destieno, muestranos a Jesu bendicto fructo de tu vientre. $O$ clementissima. O piadosa. $O$ dulce virgen Maria. Uer[sus]. Ruega por nos sancta madre de dios. Resp[onsus]. Que seamos dignos de las promissiones de Christo. Amen.

\section{(XXVI) 1577:}

SAlue te Dios Reyna y madre de misericordia, vida y dulçura esperança nuestra. Dios te salue a ti llamamos los desterrados hijos de Eua. A ti sospiramos gimiendo y llorando en aqueste valle de lagrimas. Ea pues abogada nuestra buelue a nosotros essos tus misericordiosos ojos, y despues de aqueste destierro, muestranos a Jesu bendicto fructo de tu vientre. $\mathbf{O}$ clementissima. $O$ piadosa. $O$ dulce virgen Maria. Uer. Ruega por nos sancta madre de dios. Resp. Que seamos dignos de las promissiones de Christo. Amen.

Ripalda 1591, en una columna, que aquí marcamos con (l), intercalado con (XXVII) 1584:

DIos te salue [XXVII SAlve te Dios] I Reyna [XXVII r] y madre I de misericordia [XXVII ,] I Vida [XXVII ,] y [XXVII Ø] [XXVII ,] dulçura [XXVII,] I y esperança nuestra, [XXVII Ø] । Dios [XXVII d] te salue [XXVII v]. I A ti llamamos I los desterrados I hijos de Eua [XXVII v]. [XXVII :] I A ti sospiramos [XXVII ,] I gimiendo y llorando I en este valle I de lagrymas [XXVII i]. I Ea pues I abogada nuestra [XXVI ,] I buelue [XXVII v] a nosotros I essos tus ojos I misericordiosos. I Y despues deste destierro I muestranos a IESVS [XXVII Iesus] I fructo benditol de tu vientre. | O clementissima, [XXVII .] । o [XXVII O] piadosa, [XXVII .] । o [XXVII O] dulce Virgen [XXVII v] Maria. I Ruega por nos I santa madre de Dios [XXVII ,] I porque [XXVII para que] seamos dignos I [XXVII de alcançar ] delos prometimientos [XXVII las promessas] I de IESV [XXVII Iesu] Christo [XXVII c] I [XXVII .] Amen Iesus [XXVII Ø].

(XXXII) 1590 [de Pedro Simón Abril] Salutaçion de la santa Iglesia a la santissima Virjen.

DIos te salve reyna madre de misericordia vida, dulçura, $i$ esperança nuestra. Dios te salve. A ti damos bozes los desterrados hijos de Eva: a ti suspiramos jimiendo i llorando en este valle de lagrimas. Ea pues abogada nuestra buelve nos essos tus ojos de misericordia muestranos despues deste destierro a jesus bendito fruto de tu vientre, o benina, o pia o dulçe siempre virjen Maria. Ruega por nos santa madre de Dios, que seamos hechos dinos de las promessas de Cristo. Amen. 
(XXXIII) 1596:

DIos te salue reyna y madre de misericordia. Vida y dulçura, y esperança nuestra, Dios te salue. A ti llamamos los desterrados hijos de Eua. A ti sospiramos gimiendo y liorando en este valle de lagrimas. Ea pues abogada nuestra, buelue a nos aquessos tus ojos misericordiosos. $Y$ despues deste destierro muestranos a Jesus bendito fruto de tu vientre. $O$ clementissima. $O$ piadosa. O dulce virgen Maria. v/. Rogad por nos santa madre de Dios. R/. Porque seamos dignos delos prometimientos de Jesu Christo. Amen.

Adelantamos algunas consideraciones en cuanto al contenido y al estilo de conjunto: como antífona la salve se presenta como una oracion comunitaria. Al nombrar a Maŕa como medio para la salvación en su papel de intercesora, y a Eva, implícitamente, como causa del destierro y perdición (los orantes se definen como sus «desterrados hijos»), la salve condensa la historia de los dos Testamentos.

De entrada hemos de reparár en el sentido de $s a l(u) e$, que ya nos ocupó en nuestro análisis del avemaría ${ }^{15}$; allí dejamos sin resolver el problema de cast. medieval saluar como saludar o como salvar, y la resolución semántica en uno u otro sentido (v.i.); de la Cartilla (XXXII) 1590 podemos agregar ahora un párrafo significativo, por cuanto, tras presentar la salve como antífona, puntualiza:

La salutacion del Anjel ala santissima Virjen, i la dela santa Iglesia las avemos dexado assi en sus primeras palabras, por estar assi reçebidas de muchos años atras. Pero es cosa çierta, que conforme al testo Griego, i a la traduçion Latina, i a la dotrina e interpretaçion de los santos Teologos antiguos, avia de dezir, Gozate Maria toda graçiosa. Porque aquella manera de salutaçion, Dios te salve, es mas acomodada para los pecadores,

donde el autor remite a gr. jaire para el saludo, y aparta la letra de Dios te salve como solidaria con los pecadores.

Corominas en $D C E C H$ s.v. salvo pasa sin sentir a saludar, derivando el sentido de Dios te salve. Autoridades, sin embargo, s.v. salve interpreta:

Voz latina que vale Dios te guarde, y se usa en nuestro Castellano en la misma significación ${ }^{16}$.

15 «El avemaría en la enseñanza de la doctrina cristiana entre c. 1496 y $1596 \%$, en Pulchre, bene, recte, Estudios en Homenaje al prof. Fernando Gonzalez Olle, ed. Carmen Saralegui y M. Casado, Pamplona, Universidad de Navarra, 2002, págs. 971-80; v. pág. 975 y n. 12.

${ }^{16}$ Incidentalmente valga recordar a Alonso de Palencia, quien s.v. salue se atiene a los significados del latín clásico. «Salue saluete salueto es verbo con que uno a otro denuncia salud, non tiene más tiempo y es verbo de la segunda conjugación. Salue viene de salud quiere dezir "buena salud ayas'. Dende viene saluo, as. Es saluare fazer a otro saluo. Quito de peligron; siguen las definiciones de salus, salutaris, salutatorium, salutare sin referencia alguna a contextos cristianos. Véase también la definición totalmente "clásica» de virgen (citamos por la ed. fcs. de Sevilla, 1490, alí publicada por la Asociación de las Academias de la Lengua Españolan, Madrid, 1967). 
En el Fuero de Alcala se da con frecuencia la combinación de saluar y saludar: «...e los otros saluense con .xij. xij. $\tau$ saludenlos» ${ }^{17}$. En una canción de la que citaremos del Cancionero de Baena (Madrid 1993), J. González Cuenca y B. Dutton no dudan en transcribir el saludo del ángel con «salvo a ti», aun cuando el texto reza «saluo a ty». Quedan, pues, cosas por ver.

Al texto en sus manifestaciones algo distintas entre sí aplicaremos nuestro esquema, que se presta para el ejercicio de poner cosa con cosa (aun cuando la gramática, y en general la lingiística, vaya cambiando y actualizando la clasificación de «la cosa»). Consideraremos, pues, (A) el texto, (B) lo que refleja de la prosa métrica a que aludimos, (C) el tipo de letra y la grafía y fonética, (D) la morfosintaxis, (E) el léxico, (F) la estructura retórica, más (G) un juicio sobre la traducción; todo ello ajustado a una plegaria breve, que justifica la concisión de los apartados; agregamos (H) para la iconografía. Para la comparación de la salve en otros idiomas nos limitaremos aquí al texto inglés.

A.-En cuanto al tenor del texto, la errata más extensa y significativa la representa la introducción de «después que de aqueste destierro seremos salidos» en (III) 1526 a continuación de «bendito fruto de tu vientre», tras haber escrito «y después de aqueste destierro muestra nos a Jesu», que podría reflejar dos maneras de rezar la salve en la recitación mental o una glosa de dicho tramo. En el texto latino de (XII) 1564 se da la inversión errónea «virgo semper Maria»; en las Cartillas castellanas (I-II-V) la omisión de madre, reemplaza el título usual de madre de misericordia con el de reina de misericordia ${ }^{18}$. En las Cartillas (I-II) «dulçor de vida», al no ajustarse al texto latino, siquiera cual to reflejan las Cartillas, representa una interpretación feliz que la reimpresión (V) de c. 1539 enmienda con «Dulçor vida", to que no impide leer seguidos los tres atributos: «dulçor, vida, esperança nuestra» por cuanto la ausencia de (,) es característica de la puntuación de la época, según se dirá.

C.-El tipo de letra gótica de las Cartillas (I-II-V) se prosigue en todas menos en las (XVI) 1156 y (XXXII) 1590, en cursiva, y la (XXVII) 1584, en letra redonda. Las Cartillas (I y II) llevan inicial de mayor a menor con adorno de rasgueo:

17 Cf. M." Jesús Torrens Álvarez, Edición y estudio lingtirstico del Fuero de Alcalá (Fuero Viejo), Alcalá, Fundación Fuero del Rey, 2002, pág. 376, donde la autora del estudio transcribe salvense, pág. 466.

18 Mater habfa sido una interpolación temprana, que a estas alturas no sabemos si cuenta como tal. 
1.1. Las abreviaturas y compendios del texto latino son las consagradas ya en latín, con el signo tironiano, el de versículo y el de su respuesta, representados respectivamente:

$$
\text { signo tironiano: } z \text { versículo: } \quad \text { respuesta: } B \text {, }
$$

que aquí representaremos con $\tau$, v/ y R/. Para Christi el compendio es rpji .

De las del texto castellano señalamos que la lucha entre sancto y santo se resuelve a favor de aquél en las Cartillas (I) 1496, (III) 1526, (V) c. 1539, (VIII) 1545, (X) 1554, (XI) 1558, (XVI) 1566, (XVII) 1569, (XXII) 1573, (XXV) 1576, (XXVI) 1577; de éste en las Cartillas (II) c. 1501-1508, (XXVII) 1584, Ripalda 1591, (XXXII) 1590, (XXXIII) 1596.

1.2. Se une la preposición con el adjetivo demostrativo en (I-II-V) 1496, c. 1501-1508, c. 1539, (III) 1526, Flórez 1552, (X) 1554, (XI) 1558, (XVI) 1566, (XXII) 1573, (XXVII) 1584, Ripalda 1591, (XXXII) 1590, (XXXIII) 1596, deste; pero no el pron. átono con el verbo en Ripalda «buelve nos...». También aparece en un solo tramo el nombre salueregina en (XXV-XXVI), (XXXIII).

1.3. La mayúscula (M) marca el exordio (SAlue), y el principio de los tramos, e incluso Dulçor y Esperança en las Cartillas (I-II-V); de los tramos principales en la (III) 1526: pero allí en los nombres propios no en maria, ni en madre de dios, pero sí en Eva y en Jesu; lo que se repite en la (X) 1554 excepto en jesus.

1.4. La tilde vimos que es de imprenta sin efecto fónico en la Cartilla (XXII) 1573, y como signo diacrítico se da en la Cartilla (XXXIV) pero irregularmente; en la Cartilla (VI) 1566, castellano-arábiga, es constante como medio de transcripción. Para marcar el acento tónico no lo hemos observado en ninguna, prescindiendo de la que podría coincidir con el carácter algo irregular de la $i$.

1.5.1. El presentarse el texto a toda plana o en columnas surte efecto en la puntuación y en las abreviaturas, por lo que recordamos que la salve se imprime a renglón seguido en las Cartillas (I-II-V) 1496, c. 1501-1508, c. 1539, (III) 1526, (VIII) 1545, Flórez 1552, (XVI) 1566, (XVII) 1569, (XXII) 1573, (XXV) 1576, (XXVI) 1577, (XXVII) 1584, (XXXII) 1590, (XXXIII) 1596; a dos columnas, en las Cartillas (X) 1554, (XI) 1558; en la del P. Ripalda, en una columna sangrada respecto a los márgenes, para una mayor legibilidad. La distribución del texto en columnas o columna contribuiría a marcar el sonsonete; en ambos casos no sin sugerir el canturreo. 
1.5.2. Los signos de puntuación merecen observarse como hábito paleográfico y/o de la supuesta relación con el ritmo, al que acabamos de aludir. Nos limitaremos como representativa a la invocación final; allí se observa como más o menos persistente la puntuación con (.) ante la interj. (O[h]): en latín, en las Cartillas (I-II-V) 1496, c. 1501-1508, c. 1539, (VIII) 1545 «O clemens. $O$ pia. $O$ dulcis virgo Maria»; y también en Flórez (con la errata clemenes), y en las (IX) 1549 y (XII) 1564 (en ésta con la inversión errónea «virgo semper Marias); en castellano, con el signo (:) y la interjección con mayúscula o minúscula en las Cartillas (I) c. 1496, (V) 1539 y (XXII) 1573 «O clemente: o pia. $O$ dulce virgen maria.»; con omisión de uno de los (.) en la Cartilla (II) c. 1501-1508 «O clemente $O$ pia. $O$ dulce virgen maria.», y en las (III) c. $1526 \ll O$ mansa. $O$ piadosa $O$ dulce virgen maria»; (VIII) 1545 (en columna) «O clementíssima. O piadosa o dulce virgen Maria»; en (X) 1554 (también en columna), con la interjección regularizada con $(\mathrm{M}) \ll O$ clementisima. $O$ piadosa. $O$ dulce siempre virgen maria»; sin signo de puntuación intermedio en (XI) 1558 «O clementissima o piadosa o dulce siempre virgen Maria»; (XVII) 1569 vuelve al (.) «O clemente. $O$ piadosa. O dulce virgen Maria»; que nos inducen a suponer que la interj. $o(h)$ pide una pausa, al igual de lo que acabamos de ver acerca de la conj. $Y$, precedida por (.), (:) o (E), pero sin prejuzgar necesariamente la ausencia de pausa.

1.5.3. En la grafía una tilde (como trazo llano) forma parte de la representación de la $\langle\dot{i}\rangle$, de imprenta, constante en la Cartilla (XXXIII) 1596, y alternando con $\langle i\rangle$ sin tilde en la misma palabra en la Cartilla (XXII) 1573, e.g. en miserícordiosa. Se da como signo diacrítico en la prep. $a$ en la Cartilla (XXXIV) 1536.

2.1. La $<\mathrm{u}>$ representa la vocal, y también la consonante como intervocálica en salue, eua en todas; como explosiva aparece en aduogada en la Cartilla (X) $1554^{19}$; <y> representa la vocal $i$ en el diptongo descendiente en reyna; también como grafía culta propia de Ripalda en lagrymas. Responden al valor fonético bilabial sonoro la $\langle\mathrm{b}\rangle$ inicial en buelue, como también alí la $\langle u\rangle$, pero no la explosiva $\langle u->$. La fricativa intervocálica se representa con <-u-> en todas menos en las Cartillas (XXVII) 1584, (XXXII) 1590, eva, con lo que el cambio de eua a Eva no puede atribuirse más que a las convenciones editoriales a las que acabamos de aludir, tal vez para alertar a los lectores acerca del personaje bíblico. La forma aduogada que acabamos de citar como de la Cartilla (X) 1554 representa un compromiso entre la forma latina y la ver-

\footnotetext{
19 Para estas citas hay que tener en cuenta las fuentes: para Berceo Loores v. 220d: «reina coronada de tan noble corona a ti facemos abogadan la ed. de 1780, y para la cita que adujimos arriba del Setenario, unos manuscritos posteriores al original.
} 
nácula. El grupo latinizante -ct- que vimos en sancto abreviado se da en fructo en las Cartillas (V) c. 1539, (VIII) 1545, (X) 1554, (XI) 1558, (XVI) 1566, (XVII) 1569, (XXII) 1573, (XXV) 1576, Ripalda 1591, frente a fruto en (I) 1496, (II) c. 1501-1508, (III) 1526, (XXVH) 1584, (XXXII) 1590, (XXXIII) 1596. El grupo culto $-g n-$ en el responsorio se da en todas menos (I-II-V) 1496, c. 1501-1508, c. 1539 y (XXXII) 1590 de Pedro Simón Abril.

Observamos la alternancia de la inflexión: en las Cartillas (I-II) 1496, c. 1501-1508, gemiendo y (V) c. 1539, (III) 1526, (VIII) 1545, Flórez 1552, (X) 1554 y (XI) 1558, (XVI) 1566, (XVII) 1569, (XXII) 1573, (XXV) 1576, (XXVII) 1584, Ripalda 1591, (XXXIII) 1596, gimiendo; en (XXXII) $1590 \mathrm{ji}$ miendo; $\mathrm{y}$ en (I-II-V) 1496, c. 1501-1508, c. 1539, (XXVII) 1584, (XXXII) 1590 , suspiramos y sospiramos en (III) 1526, (VIII) 1545, Flórez 1552, (X) 1554, (XI) 1558, (XVI) 1566, (XVII) 1569, (XXII) 1573, (XXV) 1576, (XXVI) 1577, Ripalda 1591, (XXXIII) 1596 (para la alternancia desde Cid sospirar, pero también sospiro y tanto suspirar como sospirar en el Quijote cf. DCECH).

2.1.1. Del nombre Jesús tenemos las representaciones siguientes, con Jesu por fonética sintáctica ante (c)risto; entre uno y otro jesu en (I) 1496, ([II] iesu) c. 1501-1508, (V) c. 1539, (III) 1526, (VIII) 1545, (XVII) 1569, (XXII) 1573, (XXV) 1576, (XXVI) 1577; Jesus en Flórez 1552, (X) 1554, (XI) 1558, (XXXII) 1590, (XXXIII) 1596; Jesu cristo por segunda vez en Flórez 1552, (X) 1554, (XI) 1558, (XXXIII) 1596; lesus (XVI) 1566, (XXVII) 1584, Ripalda 1591; Christo lesu en (XVI) 1566; Iesu Christo (XXVII) 1584, y Ripalda 1591. El fonema prepalatal africado sonoro rehilante aparece representado con $<\mathrm{j}>$ en jimiendo (XXXII) 1590.

2.2. En la prosodia Jesu cristo, que acabamos de ver en Flórez 1552, (X) 1554, (XI) 1558, (XXXIII) 1596, no estorba que el nombre se leyera con apoyo en (c)risto, pero sin impedir la inversión que también vimos en (XVI) 1566 Christo lesu. En el nombre de la salve (v.i. E 0), la conservación en el original latino regina, por el cursus planus que forma con salve, demuestra que se pronunciaba ya reina, y no reína, que hubiese producido el ritmo deseado en la lengua vernácula.

D1.-En la morfosintaxis merece especial atención de misericordia en la mayoría de las Cartillas en lugar del adjetivo misericordioso; aquél junto a reina como calco, a través del texto latino, del casus constructus que en hebreo suple a la escasez de adjetivos; aún más cerca de la matriz semítica calcada al latín está «Pater misericordiarum» que cita Blaise ${ }_{1} \$ 141$.

2. Se observa la ausencia del artículo determinado en la variante interpretativa, que se aparta de la letra, en (I-I) c. 1501-1508; en (V) c. 1539 «dulçor de vida» (v.s.); la aposición no viene introducida con artículo en (I-II-V) 1496, 
c. 1501-1508, c. 1539, «A ti clamamos desterrados hijos de Eua»; sí con él, en (III) 1526, (VIII) 1545, Flórez 1552, (X) 1554 y (XI) 1558, (XVI) 1566, (XVII) 1569, (XXII) 1573, (XXV) 1576, (XXVI) 1577, (XXVII) 1584, Ripalda 1591, (XXXII) 1590, (XXXIII) 1596, «A ti llamamos los desterrados hijos de Eua». En inglés con la inserción de un adjetivo «poor banished children of Eve».

3. El adjetivo calificativo santo se antepone a Madre de Dios; en el caso de misericordioso, cuando éste se repite en presencia del posesivo aparece antepuesto al sustantivo en «tus misericordiosos ojos» en (I-I-V) 1496, c.1501-1508, c. 1539, (III) 1526, (VII) 1545, (XVII) 1569, (XXV) 1576; pospuesto en «tus ojos misericordiosos» en (X) 1554, (XI) 1558, (XVI) 1566, (XXVII) 1584, Ripalda 1591, (XXXIII) 1596.

4.1.1. El pronombre personal de primera persona, plural, como propio de las antífonas, tras preposición es nos «a nos» en (I-II-V) 1496, c. 1501-1508, c. 1539, (X) 1554 y (XI) 1558 y (XXXIII) 1596, "buelve nos" (XXXII) 1590 y «por nos» en (I-II-V) 1496, c. 1501-1508, c. 1539, (III) 1526, (VIII) 1545, Fl6rez 1552, (X) 1554, (XI) 1558, (XVI) 1566, (XVII) 1569, (XXII) 1573, (XXV) 1576, (XXVI) 1577, (XXVII) 1584, Ripalda 1591, (XXXII) 1590, (XXXII) 1596; es nosotros, «a nosotros», en (III) 1526, (VIII) 1545, Flórez 1552, (XVI) 1566, (XVII) 1569, (XXII) 1573, (XXV) 1576, (XXVI) 1577, (XXVII) 1584 y Ripalda $1591^{20}$.

El tuteo constante en los textos de la salve, a diferencia de otras oraciones que en el s. XVI introducen el vos, confirma que el tratamiento antiguo, desde el latín clásico, puede y debe llegar hasta el presente ${ }^{21}$.

4.1.2. Como pronombre de $1^{2}$ persona plural nos, que acabamos de mencionar, aparece por separado, lo que pone en duda su atonicidad, en (I-II-V) 1496 , c. $1501-1508$, c. 1539 «a nos demuestra», (III) 1526 , (VIII) 1545 , (X) 1554, (XI) 1558, (XXII) 1573, Ripalda 1591 y (XXXII) 1590 «muestra nos», en (XVI) 1566 más el de $3^{2}$ persona: «muestra nos le»; en Ripalda 1591 ya hemos visto «buelve nos».

\footnotetext{
${ }^{20}$ Como ya observábamos acerca del padrenuestro en el ensayo citado, pág. 486.

${ }^{21}$ Sobre el problema del tratamiento cf. la bibliografĩa que ofrece R. Lapesa en Historia de la lenga española, 95.4. Más FzR 4. págs. 58-107; vid págs. 80-85. La Gramática descriptiva de la lengua española de $\mathrm{I}$. Bosque y Violeta Demonte, Madrid, 1999, \& 2.5 (en adelante GDLE) bajo la «pragmática de los pronombres de tratamiento» no incluye las plegarias para este uso de tú y vos aun cuando vige hoy; la evolución histórica de los sistemas de tratamiento pronominales suele fecharse desde el siglo xvi con usted. Se comprende que el voseo interese más en unas investigaciones centradas en un concepto mundano de la sociedad real, pero la salve hubiera podido ser objeto del estudio de la pragmática como acto de habla, aun cuando la comunicación (cf. GDLE \$60.1.1) no es interpersonal en el plano humano.
} 
4.2. El adj. posesivo no aparece ante los títulos de la virgen, a diferencia de Ia oración en inglés, donde se repite ante cada uno de los correspondientes: «our life, our sweetness, and our hope», donde se ve más a las claras la relación objetiva de las tres conceptos con el orante. Modifica el nombre del órgano del sujeto: «buelve ... tus ojos» en todos.

4.4. En cuanto al adjetivo demostrativo, los textos de la salve ilustran la alternancia, al principio no necesariamente cronológica, entre la forma compuesta aqueste en (III) 1526, (VIII) 1545, (XVII) 1569, (XXV) 1576, y este en (I-II-V) 1496, c. 1501-1508, c. 1539, Flórez 1552, (X) 1554, (XI) 1558, (XVI) 1566, (XXII) 1573, (XXVII) 1584, Ripalda 1591, (XXXII) 1590, (XXXIII) 1596; desde el punto de mira de la persona receptora del mensaje cuando el orante pide a la Virgen que dirija sus ojos hacia el cuadra aquesse en (XI) 1558, (XXXIII) 1596 «aquessos tus ojos»; pero también se lee aquel en (I-II-V) 1496, c. 1501-1508, c. 1539, (XXII) 1573 «Ea pues abogada nuestra aquellos tus misericordiosos ojos a nos buelue». La errata en (XVII) 1569 «aquel ste» tal vez delate inseguridad entre este y aqueste.

5.1. El verbo llamar sería de por sí intransitivo como clamar y el sintag. mático dar vozes; pero aquí aparece con la $a$ ante el referente en quien recae la acción; y con más razón lleva a el verbo suspirar, cf. el texto inglés (sin alternativa en la elección del verbo to $c r y$ ): «To Thee do we cry...To thee do we send up our signs..».

En el responsorio, el verbo ser en "seamos dignos», respecto a un estado futuro, en (III) 1526, (VIII) 1545, Flórez 1552, (X) 1554, (XI) 1558, (XVII) 1569, (XXII) 1573, (XXV) 1576, (XXVI) 1577, (XXVII) 1584, Ripalda 1591 y (XXXII) 1596 alterna con la versión servil de lat. effici «seamos hechos dignos» en (XVI) 1566 y (XXXII) 1590.

5.4.1. En estas variantes aisladas acabamos de ver en ser hecho la diátesis que atribuiremos a versión servil (en algunos testimonios del Padrenuestro, hemos visto ser hecho por lat. fieri: «sea hecha tu voluntad»; cf. loc cit. pág. 461); id. en el texto inglés «that we be made worthy» con el auxiliar to make.

5.4.2. La única excepción respecto al modo indicativo la representa significativamente el imperativo en la petición final, acorde con el tratamiento familiar de tú; aparte del subjuntivo en la subordinada final del responsorio.

5.5. Entre los tiempos se destaca el futuro perfecto en las Cartillas (III) 1526, (VIII) 1545, (XXII) 1573 «después de que de aqueste destierro seremos salidos» (v.s. A). 
5.6. El paradigma del verbo preludia el regular de hoy. Pero aprovechamos este apartado para observar que en castellano salve se presenta solo en singular, frente al latín (cf. Eneida 7.121 «Saluete penates»).

6. No hay adverbios, por cuanto el adj. misericordioso, dicho de la mirada («los ojos») hace innecesario un misericordiosamente; por lo demás, el grado de insistencia lo desempeñan directamente los imperativos.

7. En cuanto al uso de las preposiciones, la prep. $a$ en «a nos buelue», en correspondencia con el original «ad nos», se da en (I-II-V) 1496, c. 1501-1508, c. 1539, y ubuelve a nosotros» en (III) 1526, (VIII) 1545, Flórez 1552, (XVI) 1566, (XVII) 1569, (XXI) 1573, (XXV) 1576, (XXVI) 1577, Ripalda 1591, (XXVII) 1584, «buelve a nos...» en (X) 1554, (XI) 1558 y (XXXIII) 1596, contrasta con su ausencia en (XXXII) 1590 «buelve nos».

8. La conjunción copulativa se observa entre los títulos de la Virgen en (X) 1554, (XI) 1558, (XVII) 1569, (XXII) 1573, (XXV) 1576, (XXVI) 1577, Ripalda 1591, (XXVII) 1584, (XXXIII) 1596; se yuxtaponen sin (,) en las demás. Se unen con la conjunción copulativa las acciones afines y yuxtapuestas de (g)emir y llorar en la subordinada implícita de gerundio; van ostensiblemente en asíndesis los atributos de la Virgen, que el ritmo staccato realza, y las acciones de ( $c l$ )amar y $\mathrm{s}(u)$ spirar, se unen por medio de la conj. copulativa el $6^{\circ}$ tramo y el $7^{\circ}$ con la petición de «bolver los ojos» hacia los sujetos orantes y mostrarles el fruto de la salvación; podríamos aducir aquí pues, si con GDLE § 58.7 lo consideramos como un conector discursivo consecutivo, o con 58.7.1 un conector deductivo, en consecuencia de los méritos reconocidos a la Virgen; y nos sería fácil clasificar así, como enfático, Ea pues, menos en (XXII) 1573 «Pues ya», donde pues antepuesto al tónico ya, se realiza como átono.

9. Como interjección además de $O(h)$ (y no $a y$, que hubiese valido para la queja) observamos $e a<$ lat. eia (cf. $D C E C H$, que señala gr. $\varepsilon t \alpha ; D E A$ s.v. ea 1 «se usa para exhortar»), del que acabamos de observar que va seguido del ya mencionado pues tónico, en correspondencia con lat. ergo (a diferencia del himno Stabat mater, donde aparece por sí, como simple exhortativo en «Eia, mater, fons amoris...»); así en todas las Cartillas, menos en la (XXII) 1573, donde se lee pues ya, con ya de por sí exhortativo (DCECH lo señala como frecuente en el Arcipreste de Talavera, donde efectivamente aparece a menudo; cf. Ralph y Lisa S. De Gorog, Concordancias del Arcipreste de Talavera [Madrid, 1978]); GDLE en \$ 63.6.5.3 ilustra ya como marcador conversacional, y por tanto distinto del que tenemos aquí, donde expresa una insistencia religiosa, aunque se tutea la persona a la que ésta va dirigida. 
10. Aparte del simple salve al principio, como oración elíptica para el saludo, e interpretado como tal al repetirse en el interior (ya ventilamos la dificultad de 'salvación' con Dios como sujeto, representa en relación con la Virgen), la salve regina consta de cinco oraciones simples (sin que los méritos que se atribuyen a la Virgen ocasionen el uso de oraciones relativas); hemos visto que Flórez 1552 introduce, poco oportunamente, una oración incidental, «pues que asi es» fol. Ixxiiij; y las Cartillas (III) 1526, (VIII) 1545, (XXII) 1573, una subordinada: «después de que de aqueste destierro seremos salidos». El responsorio, en correspondencia con lat. $u t$, contiene una oración final introducida con porque o con el simple que como oración final (cf. DRAE 16).

Se da en todas la subordinación implícita de gerundio en «a ti $s(u)$ spiramos g(i)miendo y llorando". El corto número de oraciones se compensa con las aposiciones típicas del culto mariano, y las otras dos que constituyen a los orantes en pecadores y en desterrados, aquélla como confesión, ésta, como constatación.

11. El orden del pron. personal respecto al predicado lo hemos visto recto o inverso en los apartados 4.1.2 y 7; el inverso, el de las palabras con el complemento anticipado, en (I-II-V) 1496, c. 1501-1508, c. 1539, «A ti clamamos desterrados hijos de eua. A ti suspiramos», y en «aquellos tus misericordiosos ojos a nos buelue. $\mathrm{Y}$ a jesu cristo bendicto fruto de tu vientre: despues deste destierro a nos demuestra».

12. La aparente regularidad sintáctica resulta de un compromiso entre el castellano y el latín, afirmándose éste en el orden de las palabras que acabamos de ilustrar; en lugar de «Dios te salve» de la mayoría tenemos «Salve te (D)ios» en (III) 1526, (VIII) 1545, (XVII) 1569, (XXV) 1576, (XXVI) 1577.

E.-Son tres los nombres propios: (M)aria, que emerge al final, Jesus y Jesu (c)risto, también Christo lesu en (XVI) 1566 (v.s.) y Eva. A María se la llama Virgen y reina (que se emplea hoy como nombre hipocorístico para dirigirse cariñosamente a las niñas, a la par que rey para los niños; por lo que el nexo con nuestra oración queda sub iudice); en todo caso el texto inglés «Hail holy Queen» es significativo por el uso modificado de 'reina'. En lugar de «san(c)ta madre de Dios» en el responsorio Flórez la llama «virgen madre de Dios». Se realiza sal( $u)$ e regina como sustantivo en el nombre de la oración, mencionado en forma explícita en las Cartillas (I-II) \&Y seria bien que supiessen y rezassen esta oración de nuestra señora que se llama sal(u)e regina», y en la Doctrina del P. Florez: «Entre las oraciones devotas ordenadas en la yglesia: y muy acostumbrada es la Salue regina» fol. lviii *v-lix; también en el título de la oración cuando lo hay; a saber: (VIII) 1545 La salue. Regina en romance, (X) 1554 La Salue, (XI) 1558 La salue regina, (XVII) 1569 La salue 
regina en romance, (XXI) 1573 íd, con (M), (XXV-XXVT) 1576-1577 La Salueregina en Romance, y con (m) (XXXIII) 1596 La salueregina en Romance; por lo que es conocida hasta hoy por el exordio latino, como el avemaría, el credo, y el paternóster (pero también padrenuestro).

1.1.1. Por «familias de palabras» de la misma raíz pueden agruparse el sust. misericordia, «reyna de misericordia» (I-II-V) 1496, c. 1501-1508, c. 1539, o «madre de misericordia» en todas, «ojos de misericordia» en Flórez 1552, (XXII) 1573, (XXII) 1573, (XXXII) 1590, y el adj. misericordioso en (III-V) 1496 , c. $1501-1508$, c. 1539 , (III) 1526 y (VIII) 1545 , (X) 1554 y (XI) 1558, (XVI) 1566, (XVII) 1569, (XXV) 1576, (XXVI) 1577, (XXVII) 1584, Ripalda 1591, (XXXIII) 1596; y en (XXII) 1573 «o misericordiosa Maria». Salve pertenece al tema de la salvación como base de nuestra plegaria.

1.2. Se repiten dentro de un breve espacio sal(u)e, como exordio, y en la forma explícita de saludo en Dios te salue (en Ripalda por dos veces); también madre, menos en las Cartillas (I-II-V) 1496, c. 1501-1508, c. 1539, donde se omite (v.s. A), primero por lat. mater y luego por lat. genitrix del responsorio (v.i.), con lo que allí tenemos un solo lexema por dos del original.

1.3. A pesar de lo que acabamos de ver de lloroso en «lloroso valle» por «lacrymarum vallis», en la salve predomina el sustantivo, como en general en las apelaciones a la Virgen por calco del hebreo asumido por la Vulgata, según se dijo: en «madre de misericordia»; de ojos en cambio se dice en todas que son misericordiosos; «ojos de misericordia» aparece solo en las Cartillas (XXII) 1573 y (XXXII) 1590; sería de este tipo «Dulçor de vida», que hemos calificado de errata. El adjetivo epíteto bendicto aparece como en latín anticipado al nombre en (I-II-V) 1496 , c. 1501-1508, c. 1539, (III) 1526, (VIII) 1545, (XVII) 1569, (XXII) 1573, (XXV) 1576, (XXVI) 1577, (XXXI) 1590, (XXXIII) 1596, y no en Flórez, (X) 1554, (XI) 1558, (XVI) 1566, (XXVII) 1584, Ripalda 1591 «fructo bendito». El adjetivo predicativo digno $+d e$ introduce el sustantivo en la petición final: «que seamos dignos de las promissiones (o promessas o prometimientos) de Cristo», v.s. y no «de lo que Cristo nos ha prometido».

2.1. En la formación de los sustantivos carecen de moción de género madre y virgen, pero acerca de éste M. ${ }^{a}$ Teresa Echenique Elizondo señala las formas femeninas birgina, y también erregina 'reina', como excepcional en el vascuence, que no tiene moción de femenino ${ }^{22}$. En la derivación alternan los

${ }^{22}$ En la rota «Algunas consideraciones sobre conexiones románicas varias en la configuración del léxico vasco», en Pulchre, bene, recte. Estudios en homenaje al prof. Fernando González Olle, págs. 449-64 (vid. pág. 453). 
sufijos -or en (I-II-V) 1496, c. 1501-1508, c. 1539, Flórez 1552, (XXII) 1573, dulçor, y -ura en (III) 1526, (VIII) 1545, (X) 1554, (XI) 1558, (XVI) 1566, (XVII) 1569, (XXV) 1576, (XXVII) 1584, Ripalda 1591, (XXXII) 1590, (XXXIII) 1596, dulçura (por eufonía no se da dulceza); con ritmo diferente como se ve por el ritmo de la errata «dulçor de vida» (v.s.), siendo dulçura, aparte de la consonancia, más difundido en la prosa; alteman promission en (III) 1526, (VIII) 1545, (XVII) 1569, (XXII) 1573, (XXV) 1576, (XXVI) 1577, prometimiento en Flórez 1552, (X) 1554, (XI) 1558, Ripalda 1591, (XXXIII) 1596 y promessa en (XVI) 1566, (XXVII) 1584, (XXXII) 1590 (piénsese también en la anterioridad de defensión respecto a defensa). La formación del adjetivo se produce con el suf. -oso en lloroso de (I-II-V) 1496, c. 1501-1508, c. 1539 «en este lloroso valle» $\leftarrow$ «in hac lacrymarum valle»; en los verbos se observa el pref. de- en demostrar de las Cartillas (I-II-V) 1496, c. $1501-1508$, c. 1539 , y luego mostrar (cf. DEA smostrar 2 hacer ver [algo abstracto $c d$ a alguien]») en todas las otras, con el mismo sentido. Observamos también el verbo simple bolver como más corriente que rebolver, que también era posible como en italiano rivolgere ${ }^{23}$, con agregada explícitamente la intencionalidad ${ }^{24}$; respecto al latín con paso de verbo compuesto a verbo simple, y eventualmente a composición distinta. Otro íter es el de desterrado como participio pasivo de desterrar respecto a lat. ex(s)ul.

2.2. Además de salve ha pasado al castellano también vale con base latina; véase en Eneida 11.97 «Salue aeternum mihi, maxume Palla / aeternumque uale», pero no ave.

3.1. De sal(u)e hemos dicho que es un saludo en latín clásico (v.s. Alonso de Palencia); y así también en el bíblico; cf. 2 Re "Salve rey»; también se había trasformado en el culto en una fórmula de alabanza, como ave aplicado a la Virgen, pero no exclusivamente a persona; Blaise $e_{1}$ menciona en $\$ 35$ «Salve, latus Salvatoris», y el Lexikon fir Theologie und Kirche» cita «Salvete, Christi vulnera".

Como para oculus, ya en latín clásico y bíblico, tenemos el nombre del órgano por el de la función: «bolver uno sus ojos», 'volver o dirigir la mirada'.

En el aspecto de la extensión semántica hemos de señalar el adj. pio, que en la salve tiene el sentido de 'devoto' afín al de misericordioso ${ }^{25}$. En cuanto

${ }^{23}$ Cf. en Fr. Luis de León cuando traduce Horacio 4,1.10-12, donde el poeta se dirige a Venus: «jVete en buen hora; / revuelve alla tu llama / sobre la gente moza!».

${ }^{24}$ Frente a volver en M. Seco, Diccionario del español actual (DEA) 9 b «después se volví a stu compañero".

${ }^{25}$ Sethalé la diferencia entre este sentido tradicional y el innovativo en orden a la religión interior en «iDevoción o piedad? Apuntaciones sobre el léxico religioso de Alfonso y Juan de Valdés», Revista Portuguesa de Filologia, 7, 1956, 365-88. 
a las alternativas entre la extensión de llamar respecto a la de clamar, la familiaridad con la oración latina tendería a asimilarlos en la conciencia del orante (DCECH da clamar como forma normal en Berceo, dentro del vocabulario latinizante de este poeta, y también la señala como del Fuero de Avilés); en todo caso es bueno recordar que, según señala Blaise ${ }_{1} \S 63$, en las plegarias latinas el adj. pius es más frecuente que bonus. Volveremos sobre ambos al comparar las voces patrimoniales con los latinismos (v.i. 5).

3.3. La solidaridad del concepto de misericordia con la Virgen está moldeada con el atribuido a Dios o Señor desde el Antiguo Testamento; e.g. en Núm 6,26 «convertat Dominus vultum suum ad te», o en Ps 32,22 «fiat misericordia tua, Domine super nos» (v.q. Blaise ${ }_{1} \$ \$ 45$ y 55).

4. Son afines piadoso en la acepción que diremos, y misericordioso (vi. 5).

4.1.1. Como forma alternativa del sustantivo en «de misericordia» hemos visto misericordioso.

4.1.2. Forman serie homogénea los verbos $s(u)$ spirar, gemir y llorar, (cl)amar y (ospirar), y los adjetivos clemente, pía o piadosa y dulce; en cambio, entre dulçor o dulzura, y esperança la proximidad relativa puede compararse con la de las advocaciones de las letanías.

4.1.4. Se complementan reina, madre y abogada; éste como suficiente para implicar la intercesión de María (frente a inglés; «most gracious Advocate»).

4.2. El campo semántico es el de la intercesión, aun cuando no hay un verbo específico para ello, sino la mención del papel de abogada, que acabamos de constatar.

5. La lucha entre la palabra latinizante y la forma vemácula se manifiesta ya en la grafía en aduogada (II) c. 1501-1508, (X) 1554, abogada en (I-V) 1496, c. 1539, (III) 1526, (VIII) 1545, Florez 1552, (XI) 1558, (XVI) 1566, (XVII) 1569, (XXII) 1573, (XXV) 1576, (XXVII) 1584, Ripalda 1591, (XXXII) 1590, (XXXIII) 1596; en el ámbito del adjetivo, entre pio, minoritario en las Cartillas (I-II-V) 1496, c. 1501-1508, c. 1539, (XXXII) 159 y piadoso en las (III) 1526, (VIII) 1545, (X) 1554, (XI) 1558, (XVI) 1566, (XVII) 1569, (XXII) 1573, (XXV) 1576, (XXViI) 1584, Ripalda 1591, (XXXIII) 1596, aquél es favorecido por el modelo latino y también por el ritmo, como más breve y mejor amalgamado en las series; lo veremos sin castellanizar en la copla de Álvarez de Villasandino en CBaena; en el ámbito del verbo, en clamar en las Cartillas (I-II-V) 1496, c. 1501-1508, c. 1539 «a ti clamamos», y llamar en (III) 1526, (VIII) 1545, Flórez 1552, (X) 1554, (XI) 1558, (XVI) 1566, 
(XVII) 1569, (XXII) 1573, (XXV) 1576, (XXVII) 1584, Ripalda 1591 y (XXXIII) 1596, sin que se pueda determinar una diferencia semántica; en (XXXII) 1590 «A ti damos bozes», dar bozes es el equivalente vernáculo, que parte de la materialidad de voz como 'grito'. En cuanto al concepto de interce$d e r, \tan$ fundamental en la relación de los fieles con María, y en general de los cristianos con Dios, $D C E C H$ solo cita el verbo, y el sustantivo intercesión de pasada s.v. ceder, significativamente tardío (cf. interceder en Núñez de Toledo, 1499), aunque importe para la introducción de los cultismos en la lengua religiosa.

F 1.-Pudo haber motivos fonéticos de aliteración de la oclusiva labial.

2. La salve contiene una secuencia de tramos bien definidos en que se manifiesta el paralelismo propio de los Salmos y de otros textos bíblicos, impetratorios en este caso.

3. Como metonimia se conserva valle $<$ vallis para aludir al mundo o al 'más acá', contrapuesto a la gloria, que aquí no se nombra, pero está implícito en el concepto de 'salvación'.

4. Por la convivencia del castellano y el latín, a la que nos referíamos arriba, es por lo que la salve en castellano se distingue de otros idiomas, no digamos ya del inglés y del alemán, sino incluso del francés, que ha desarrollado sauver, mientras que "el tratamiento de al en salve (...tomado de lat. salve, imperativo de salv-re, 'estar en buena salud'...) indica evolución semiculta, o predominio de las clases superiores", según indica Corominas en $D C E C H$, sin mencionar nuestra oración; en el nombre de la plegaria, según vimos, se ha afirmado lat. regina; consta por igual de palabras patrimoniales y latinismos: aparte de salue, de aquellas es manso; en todas rogar como verbo generalizado en las oraciones de intercesión, como obstáculo a la introducción de interceder (I) 1496, (XI) 1558, (XXII) 1573, (XXV) 1576; en (II-V) c. 1501-1508, c. 1539, (X) 1554, (XVI) 1566, (XVII) 1569, (XXVII) 1584, (XXXII) 1590, (XXXIII) 1596 bendicto, prescindiendo de la grafía, representa un estadio intermedio entre lat. benedictus y cast. medieval benito, con bendito en Ripalda 1591; traídos del latín son clemente en (I-II-V) 1496, c. 1501-1508, c. 1539, (VIII) 1545, (XVI) 1566, (XVII) $1569 \leftarrow$ clemens; con una inclinación marcada hacia el superlativo clementissimo ${ }^{26}$, que indica un crescendo estilístico, en (X) 1554, (XI) 1558, (XXV) 1576, (XXVII) 1584, Ripalda 1591, (XXXIII) 1596, y la adecuación a otros títulos de la Virgen (Blaise ${ }_{1} \&$ cf. 119 «Virgo purissima, sacratissima, sanctissima, benignissima»).

\footnotetext{
${ }^{26}$ No conocía este texto cuando escribí mi nota sobre «El superlativo en -Lsimo y la versión castellana del Cortesano», Revista de Filologia Española, 39, 1955, 46-60, ahora en Castiglione y Boscán, 1959.
} 
Estamos en duda entre la repetición de la palabra latina, que el orante había oído y recordaría, y lo que puede denominarse como «latinismo» por una tendencia de la lengua religiosa, en el caso de pío en (I-II-V) 1496, c. 15011508 , c. 1539, (XXXII) 1590, en cuyo lugar hallamos piadoso en (III) 1526, (VIII) 1545, (X) 1554, (XI) 1558, (XVI) 1566, (XVII) 1569, (XXII) 1573, (XXV) 1576, (XXVII) 1584, Ripalda 1591, (XXXIII) 1596, en el sentido afín a misericordioso ${ }^{27}$. Con genitrix se correspondería engendradora, raramente empleado por sí, poco apto para el estilo de la oración.

G.-Forman figura etimológica llorar y lloroso: (I-II-V) 1496, c. 15011508, c. 1539 «llorando en este lloroso valle», con la concomitante aliteración; en (I-II-V) 1496, c. 1501-1508, c. 1539 «despues deste destierro a nos demuestràs la aliteración se completa con la elección de la forma derivada (v.s.).

H.-En la Cartilla mexicana (XVII) de 1569, que es la que más abunda en ilustraciones, la salve en latín lleva intercalada la representación convencional de la huida a Egipto: San José y la Virgen a caballo con el niño, contra el fondo de las torres de Jerusalén; la Cartilla (III) 1526 está encabezada por el busto de la Virgen y Niño, ambos con halo y con una corona de las que la de la Virgen se parece a la radiada, con un asomo poco distinguible de joyas ${ }^{28}$; la (VIII) 1545, casi ininteligible, lleva la Virgen con el sol y la luna; en otras, e incluso en el becerol de 1566 , la salve lleva viñetas, todas muy toscas, relacionadas con la coronación de la Virgen.

Agregamos otros texto con una oración que nos encamina hacia las letanías y tiene en común con la salve el exordio reiterado «Dios te salue», en la de la Cartilla (V) c. 1539:

DIos te salue seruidora dela trinidad. Dios te salue hija del sempiterno padre. Dios te salue esposa del espiritu santo. Dios te salue madre de nuestro señor jesu xpo. Dios te salue hermana delos angeles. Dios te salue promission delos prophetas. Dios te salue reyna de los patriarchas. Dios te salue enseñadora delos Apostoles. Dios te salue confortadora delos martyres. Dios te salue fuente $\tau$ plenitud delos confessores. Dios te salue honra delas biudas. Dios te salue corona delas virgines $\tau$ de todos los santos $\tau$ santas: y ruego te humilmente que en mis tribulaciones me ayudes. Amen. Deo gracias.

${ }^{27}$ O sea, en el sentido devoto que consideré en «¿Devoción o piedad? Apuntaciones sobre el léxico religioso de Alfonso y Juan de Valdés», que señalé en la n. 25. En los diccionarios pío se registra por primera vez en Franciosini 1620; pío e pía. Pio, pia, cioe pietoso, pietosa; deuoto, deuota. II. Stevens 1705: Pious, Godly, Merciful. Lat. Pius.

${ }^{28}$ EI nombre de «radiada», a falta de encontrar una documentación para la nomenclatura, nos lo sugiere la Dra. Natalia Horcajo, especialista en joyería del s. XVI, pero los módulos de nuestra corona forman ángulos mucho más agudos que en el modelo que nos señala. 
Como nuestro tema ha sido principalmente la salve en castellano no estarán de más sus definiciones en los principales Diccionarios; por lo pronto el Diccionario de la Lengua Española de la Real Academia Española (DRAE) 2001 dedica a la salve esta definición, breve pero satisfactoria:

Oración con que se saluda y ruega a la Virgen Maŕa II 3. Composición musical para el canto de esta oración. Il 4. Este canto.

El Diccionario del español actual de Manuel Seco $(D E A)^{29}$, al introducir una cita de autor modemo disloca la alusión a «algunas variantes» (a no ser que se refiera a las interpretaciones musicales); a saber:

1 Oración, dedicada a la Virgen, que comienza en español con las palabras «Dios te salve, reina y madre» y en latín con «Salve, regina». Tb se da este n[ombre] a algunas variantes de esta oración. I CNavarro Perros 179: Antes ... rezaba la Salve; pero ahora no me acuerdo. 2 I Composición musical sobre el texto de la Salve (1).

¿Olvido de la salve de parte del protagonista de la obra citada, o de parte de los que han confeccionado la entrada?

Con la mirada vuelta hacia el pasado valgan como apéndice estos otros textos, de entre los muchos versos inspirados en nuestra oración: en primer lugar transcribimos tres estrofas del Cancionero musical de la corte de los Reyes Católicos $^{30}$, con rimas agudas sugeridas por el estribillo; a saber:

Reina y Madre de Dios, oíd nos.

I Oídnos, Señora, pues que nos conosçemos que si bien tenemos, qu'es por vos.

II Pues, Regina çeli. acuérdese os que, si sois Madre de Dios, sois por nos.

III Mostra te ese matrem, sumat per te preçes, pues tú las ofreçes ante Dios (fol. 12v).

\footnotetext{
29 Con Olimpia Andres y Gabino Ramos, Madrid, 1999.
}

${ }^{30}$ De la ed. J. Romel Figueras, Barcelona, 1965, vol. IV-1 y 2. 
$Y$ luego la cantiga que se lee como segunda en el Cancionero de Baena, aquí en la transcripción facsímil ${ }^{31}$ y en la edición de B. Dutton y J. González Cuenca, pág. $13^{32}$; a saber:

Virgen digna . De alabança, En ty es - mi esperança.

Santa 0 clemens o pya o dulcis - vyrgo maria tú me guarda $\cdot$ noche - dia de mal - Et de tribulança.

Aue dei - mater alma llena byen - commo la palma, torna / mi - fortuna en calma mansa, con - mucha bonança.

Invyolata $\cdot$ Permaniste quando agnus - dey paryste: faz [me] - que non byua tryste, mas ledo - syn toda errança ${ }^{33}$;
Virgen digna de alabança, en ti tengo mi esperança.

Santa! O clemens! O pía! o dulçis Virgo María!

Tú me guarda noche e día De mal e de tribulança.

Ave, Dei Mater alma! llena bien como la palma, torna mi fortuna en calma, mansa, con mucha bonança.

Inviolata permansiste quando Agnus Dei pariste; fazme que non biva triste mas ledo, sin toda errança,

y la $2^{\mathrm{a}}$ estrofa de la $1^{\mathrm{a}}$ cantiga de Alfonso Álvarez de Villasandino, que en el mismo Cancionero de Baena aparece en primer lugar como «desfecha» o 'despedida' de la que acabamos de citar, donde alude al primero de los «gozos» de la Virgen; a saber, anteponiendo el texto de la prínceps:

Plazentero - fue el primero gozo señora, que oviste quando el vero $\cdot$ mensajero te saluo - tu rrespondiste troxiste en tu Seno - vyrginal al padre - Celestial al qual - syn dolor pariste
Plazentero fue el primero gozo, Señora, que oviste; quando el vero mensajero te salvó, tú respondiste; troxiste en tu seno virginal al Padre çelestial, al qual sin dolor pariste ${ }^{34}$.

${ }^{31} \mathrm{Cf} . \mathrm{C}$. de B. Reproduced in facsimile from the Unique Manuscript in the Bibliothèque Nationale, Fonword by Henry R. Lang. Printed by Order of the Trustees, New York, 1926.

32 Donde se pueden apreciar la relación a la que ahudimos al principio entre saluar y salvar, y los problemas que surgen acerca del uso del signo de admiración.

${ }^{33}$ El pronombre átono va entre corchetes metri causa, aunque hubiese sido más feliz para el ritmo y la sintaxis la introduccion del pron. personal sujeto: «faz que yo non viva triste"

34 Dejamos para otros el paralelo entre los atributos de la Vírgen y de la dama, como Seriora de valor y otros títulos de clara raigambre trovadoresca, aquél como calco de Dona de pretz; cf. M. Raynauard, Lexique roman ou dictionnaire de la langue des troubadours. 